\title{
Modal Domains and Selectivity in Large Square Lasers
}

\author{
Liming Liu, Ziyuan Li and Haroldo T. Hattori \\ School of Engineering and Information Technology, The University of New South Wales at Canberra, Canberra \\ ACT 2601 Australia,Liming.Liu@student.adfa.edu.au,liziyuanlzy@gmail.com,h.hattori@adfa.edu.au
}

\begin{abstract}
Modes in square resonators have $\mathrm{C}_{4 \mathrm{v}}$ symmetry. A special set of modes in these resonators have odd parities and behave like whispering gallery modes. These quasi-whispering gallery modes have high quality factors and exhibit interesting features. In particular, higher order quasi-whispering gallery modes present nodes that seem to be grouped into smaller domains. We show that the removal of one of these domains does not significantly affect a particular mode but can lead to significant increment in the radiation losses of other modes. This mode selectivity process can eventually be used to develop quasi single-mode lasers.
\end{abstract}

Index Terms-Integrated Optics, Micro-resonators, Photonics, Semiconductor Lasers.

\section{INTRODUCTION}

Micro-resonators are widely used in integrated optical circuits. Key applications of microresonators include optical signal processing and filtering, development of micro-lasers and quantum computation. Their unique ability to concentrate light into small spaces allows the development of many tiny integrated optical circuits. The confinement of light into small regions can be achieved by using three physical mechanisms: photonic bandgap effect [1]-[9], excitation of plasmonic waves [10]-[13] and quasi total internal reflection [14]-[26].

In those resonators based upon quasi total internal reflection, the resonator generally assumes a known geometrical shape such as micro-disks, micro-rings, micro-triangles and micro-squares. In this particular article, the analysis of modes in a square resonator of side of $9.1 \mu \mathrm{m}$ is conducted. Microsquares have whispering gallery like-modes with odd parities with respect to the diagonals of the square [26]. These modes can have quality factors (in the range of tens of thousands) and have zero magnetic/electric field intensity along the diagonals of the resonator [24], [26].

Square resonators of small dimensions can achieve single-mode operation. However, as argued by Ohnishi et al. [27] larger area lasers can produce much higher output powers, improved thermal stability and narrower beam divergences. While observing quasi-whispering gallery modes at distinct resonant wavelengths, it was noticed that a few modes exhibit interesting field patterns and seem to be clustered in small domains that go beyond the $\mathrm{C}_{4 \mathrm{v}}$ symmetry group. The term domain refers to the case where the main field ( $H_{y}$ field in case of $T E$ modes), have regions of strong electric/magnetic fields surrounded by a boundary where the field is zero, in analogy to magnetic domains in magnetic materials. The initial concept of mode domains have been reported by Chang et al. [28]. If one of 
these domains is removed, certain modes are not strongly affected by the removal of one domain, but the remaining modes are strongly affected by the removal of this surface: their quality factors drop considerably. Since the losses of other resonant modes are significantly higher than that of the selected mode, the laser device can operate under a single lasing regime over a wide range of pump optical power, providing a quasi single-mode operation of the device.The inclusion of an air hole can also lead to a control of laser emission as reported by Dejellali et al. [29].

\section{MODAL ANALYSIS AND FIELD PATTERNS IN A SQUARE RESONATOR}

The hetero-epitaxial layered structure in which a laser device is to be fabricated is shown in Fig. 1(a). It consists of a core layer of GaAs with three $7.4 \mathrm{~nm}$ thick $I n_{0.2} G a_{0.8} A s$ quantum wells, separated by $6 \mathrm{~nm}$ GaAs confinement barriers. The thickness of the core layer $\left(h_{l}\right)$ is assumed to be $140 \mathrm{~nm}$. A high content $A l_{0.98} G a_{0.02} A s$ layer is oxidized (its initial thickness $h_{2}$ is $450 \mathrm{~nm}$ ) to provide, in conjunction with the top air layer, vertical confinement of light. The quantum wells are grown to emit light at a wavelength close to $1040 \mathrm{~nm}$. Although this epi-layer structure is primarily targeted to produce optically pumped lasers, the bottom oxidized layer could be substituted by another suitable layer (e.g. Bragg stack) to produce electrically pumped sources (however, if we partially oxidize the bottom layer, electrical current can still flow to the substrate). The oxidation of the $A l_{0.98} G a_{0.02} A s$ layer can be realized in a quartz tube that is heated at $450^{\circ} \mathrm{C}$ with a constant flow of boiling water and nitrogen. When oxidized, the refractive index of the high content aluminum layer is reduced to 1.65 . It should also be mentioned that this epitaxially layered structure supports mainly Transverse Electric $(T E)$ modes with main magnetic field component perpendicular to the plane of the device ( $y$ direction) and main electric field component in the plane of the device ( $x-z$ direction).

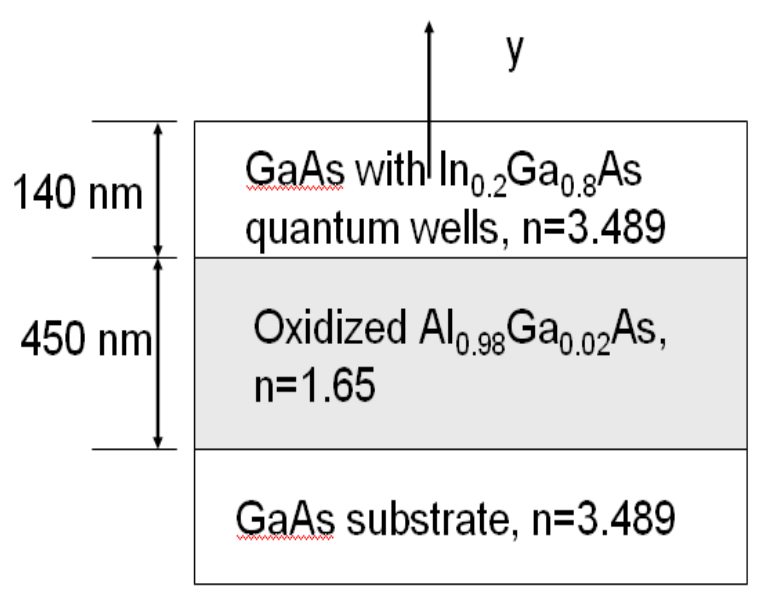

(a)

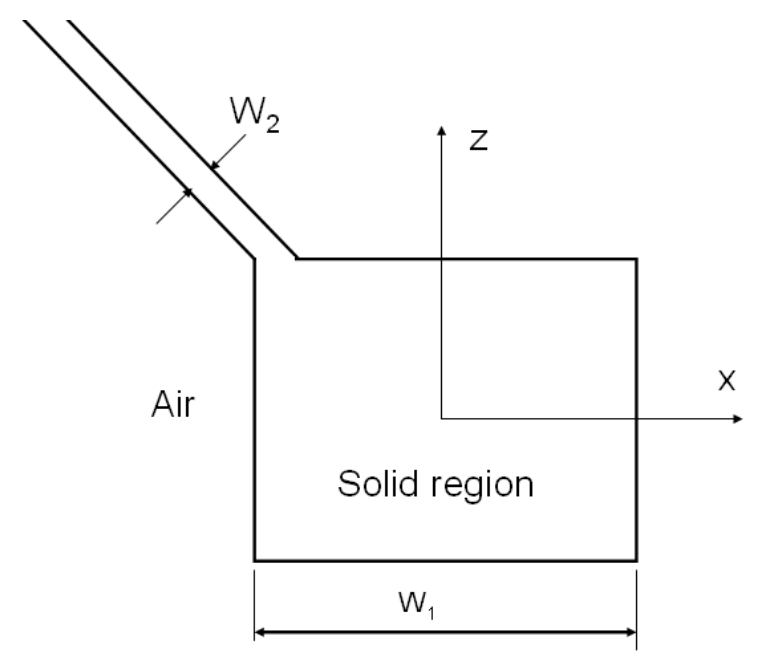

(b)

Fig.1. (a) Epitaxially layered structure (b) In-plane view of the modified square structure.

A top view of the device is shown in Fig. 1(b). The side of the square $\left(W_{l}\right)$ is chosen to be $9.1 \mu \mathrm{m}$ in order to place the desired resonant peak close to $1040 \mathrm{~nm}$. This square resonator couples light into a single-mode waveguide as shown in Fig. 1(b). The waveguide is placed close to the diagonals of the 
resonator because the modes in the square resonator have odd symmetry ( $H_{y}$ field for $T E$ modes or $E_{x}$ field for TM mode) along the diagonals of the square [26]. Hence, if we place the waveguide along this direction and displaced with respect to the diagonal (in quasi-whispering gallery modes, the electric/magnetic nodes are positioned along the diagonal directions), we can increase the coupling efficiency of the generated light into the single-mode waveguide. The single mode waveguide has a width of $400 \mathrm{~nm}\left(W_{2}=400 \mathrm{~nm}\right)$. For computational purposes, the length of the waveguide is assumed to be infinite.

To analyze these devices, commercial finite-difference time-domain (FDTD) software is employed [30]. Because of the extensive range of simulations, most of our structures are analyzed with $2 D$ FDTD simulations. The effective index of the epitaxially layered structure is $n_{\text {eff }}=2.9$ (for $T E$ modes). A few cases were further investigated with $3 D$ FDTD simulations to assess the effects of vertical losses. A source is placed at the centre of this large square resonator and is assumed to have a Gaussian spatial distribution, with a spot size diameter of $200 \mathrm{~nm}$. In case of 3D simulations, the spot size diameter in the vertical direction $(y)$ is assumed to be $80 \mathrm{~nm}$. The computation region is terminated by perfectly matching layers $(P M L)$. No material gain is added to the FDTD simulations, since parameters such as transmission through the waveguide are assessed and also because the addition of material gain may lead to numerical instabilities. The grid specified in the calculations were assumed to be $\Delta x=\Delta z=40 \mathrm{~nm}$ ( $\Delta y=20 \mathrm{~nm}$ in case of the selected $3 D$ FDTD simulations $)$ and the time step was $\Delta t=6.7 \times 10^{-17} \mathrm{~s}$. A magnetic field monitor $\left(H_{y}\right)$ is placed in the waveguide to provide the spectral response of light coupled into the waveguide. Now, in case of power budget analyses, power monitors are placed around the structure (laterally and/or vertically) and in the waveguide. For $T E$ modes, the source imposes a value of the field $\left(H^{y}\right)$ at a certain position and can be described as,

$$
H_{y}=f(t) \exp \left\{-\frac{1}{4 \phi_{\text {spot }}^{2}}\left[\left(x-x_{o}\right)^{2}+\left(y-y_{0}\right)^{2}\right]\right\}(1)
$$

where $x_{o}$ and $y_{o}$ are the coordinates of the source (the source is placed close to the side of the square) and $\phi_{s p o t}$ is the spot-size diameter of the source (it is made small to simulate a point source, being much smaller than the side of the square). The function $f(t)$ describes the temporal dependence of the source (either a sinusoidal continuous wave or a pulse with Gaussian temporal dependence).

The modes in a large square resonator are initially analyzed. Normally, the modes in a square resonator have $C_{4 v}$ symmetry that can be further sub-classified into $\mathrm{A}_{1}, \mathrm{~A}_{2}, \mathrm{~B}_{1}, \mathrm{~B}_{2}$ and $\mathrm{E}$ representations [26]. In case of $T E$ modes (main magnetic field perpendicular to the plane of the resonator), the modes can be classified as $T E_{\mathrm{ab}}$, where $a$ and $b$ denote the number of nodes in the $\mathrm{x}$ and $\mathrm{z}$ directions, respectively [26]. A special sub-class of modes that behave like whispering gallery modes and have high quality factors were further classified as $W G-T E_{m n}$ where the $\mathrm{m}$ and $\mathrm{n}$ numbers were given by [24], [26], 


$$
\begin{aligned}
& m=\frac{|a-b|}{2}-1 \\
& n= \begin{cases}a+b+4 & \text { even } m \\
a+b+2 & \text { odd } m\end{cases}
\end{aligned}
$$

In case of quasi whispering gallery $T E$ modes, the main component of the magnetic field $\left(H_{y}\right)$ along the diagonals is zero (magnetic wall). In this case, the resonant wavelengths can be determined by solving the equations [24],

$$
\begin{array}{r}
\gamma_{m}=\sqrt{2}(m+1) \frac{\pi}{W_{1}} \\
\gamma_{m}^{2}+\beta_{n}^{2}=n_{\text {eff }}^{2}\left(\frac{2 \pi}{\lambda}\right)^{2} \\
\gamma_{0}=\sqrt{\frac{\beta_{n}^{2}}{2}-\left(\frac{2 \pi}{\lambda}\right)^{2}} \\
\frac{n \pi}{2}=\frac{\sqrt{2}}{2} \beta_{n} W_{1}+2 \tan ^{-1}\left(\frac{\sqrt{2} \beta_{n} / n_{\text {eff }}^{2}}{2 \gamma_{0}}\right)+\frac{1+(-1)^{m} \pi}{2}
\end{array}
$$

where $m=0,1,2, \ldots$ and $n$ is an even number for quasi-whispering gallery modes. The parameter $\lambda$ is the free-space wavelength and $n_{\text {eff }}$ is the effective index of the slab structure. The other terms are propagation constants to be determined. For non-whispering gallery modes, $n$ can be odd. As mentioned previously, whispering like gallery modes in square resonators [24] have nulls of main component of the magnetic field (in case of TE modes) along the diagonals of the square resonator and resemble whispering gallery modes in microdisk lasers where the fields are strong close to the edges of the microdisk resonator.

Considering a basic square resonator, the magnetic field spectrum in the waveguide is shown in Fig. 2(a). There are many resonant peaks in the free-space wavelength $(\lambda)$ range between $1000 \mathrm{~nm}$ and $1100 \mathrm{~nm}$. However, the high quality factor $(\mathrm{Q})$ peaks appear at $1006.7 \mathrm{~nm}\left(\mathrm{Q}=14000\right.$, mode $\left.T E_{1,72}\right)$, $1017.3 \mathrm{~nm}\left(\mathrm{Q}=20000\right.$, mode $\left.T E_{6,72}\right), 1022 \mathrm{~nm}\left(\mathrm{Q}=20000\right.$, mode $\left.T E_{5,70}\right), 1038.1 \mathrm{~nm}(\mathrm{Q}=20000$, mode $\left.T E_{0,72}\right), 1043.9 \mathrm{~nm}\left(\mathrm{Q}=16000\right.$, mode $\left.T E_{10,68}\right), 1045.9 \mathrm{~nm}\left(\mathrm{Q}=20000\right.$, mode $\left.T E_{3,69}\right), 1064.2 \mathrm{~nm}$ $\left(\mathrm{Q}=20000\right.$, mode $\left.T E_{2,70}\right), 1067.5 \mathrm{~nm}\left(\mathrm{Q}=15000, T E_{0,70}\right), 1098 \mathrm{~nm}\left(\mathrm{Q}=20000\right.$, mode $\left.T E_{1,66}\right)$. Figure 2(b), 3(a) and 3(b) show the $H_{y}$ (y-component of the magnetic field distribution) at $\lambda=1006.7 \mathrm{~nm}, 1038.1$ $\mathrm{nm}$ and $1043.9 \mathrm{~nm}$, respectively. Based on these plots, we can clearly see that the waves have main component of the magnetic field $\left(H_{y}\right)$ with a null along the diagonals. Besides that, we can see that the mode patterns form domains in the square resonator, i.e., they are packed in small regions with positive and negative peaks with nodes between these peaks. These domains are all spread over the 
square resonator. At $1006.7 \mathrm{~nm}$, we can observe six domains along the x-direction, while at 1038.1 $\mathrm{nm}$, we can observe five of these domains. These domains are more complex at $1043.9 \mathrm{~nm}$ but we can still identify six domains along the $x$-direction. A displacement of the power source in the simulation shifts these resonant wavelengths only slightly (less than $0.5 \mathrm{~nm}$ ).

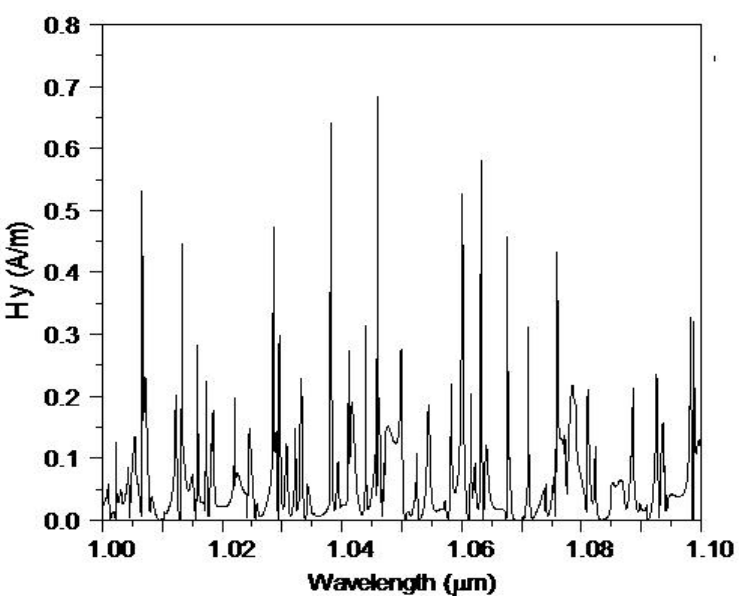

(a)

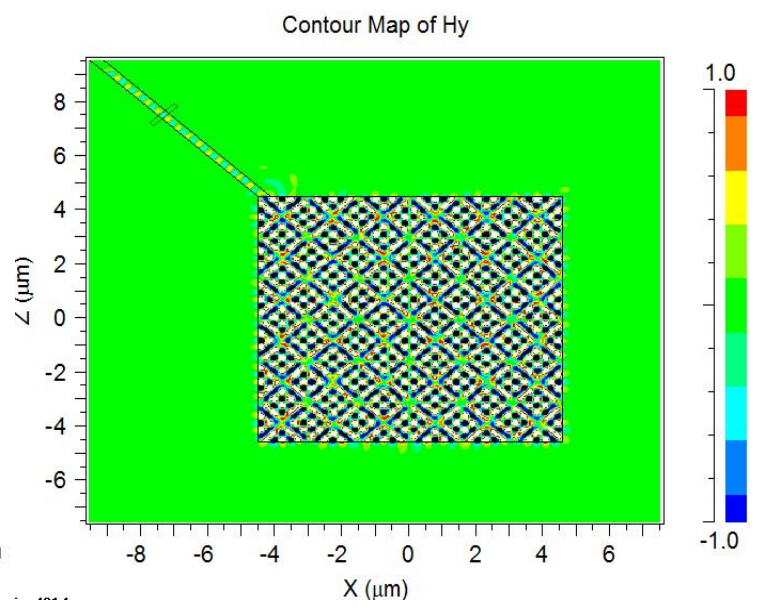

(b)

Fig. 2. Basic square resonator of $9.1 \mu \mathrm{m}$ : (a) Magnetic field spectrum (Hy) at the center of the waveguide. (b) Magnetic field (Hy) distribution at $\lambda=1006.7 \mathrm{~nm}$.

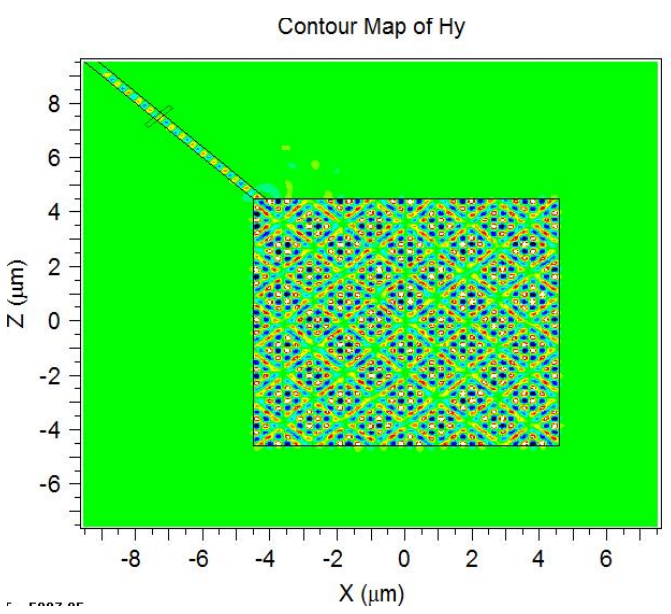

(a)
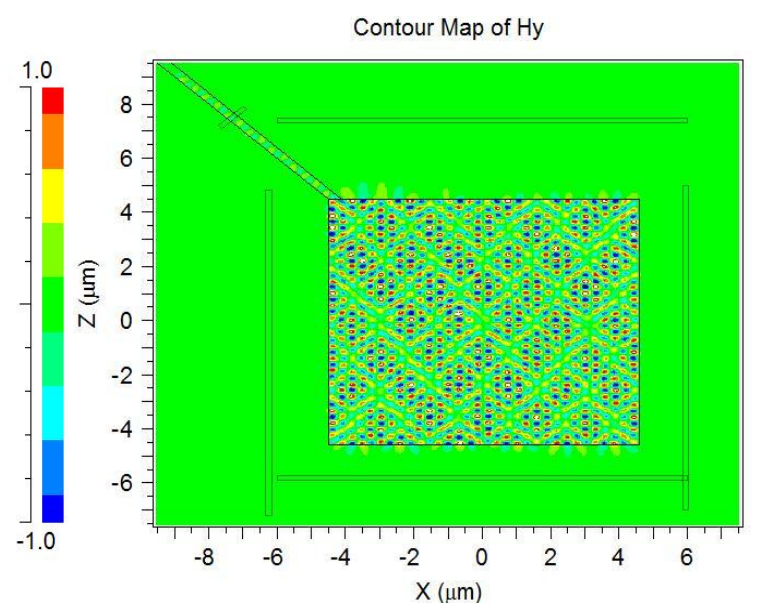

(b)

Fig. 3. Basic square resonator of $9.1 \mu \mathrm{m}$ : (a) Magnetic field (Hy) distribution at $\lambda=1038 \mathrm{~nm}$. (b)

Magnetic field (Hy) distribution at $\lambda=1043.9 \mathrm{~nm}$.

In order to assess vertical losses, we launched $3 D$ FDTD simulations. The peaks at $1006.7 \mathrm{~nm}$ (mode $T E_{1,72}$ ), $1038.1 \mathrm{~nm}$ (mode $\left.T E_{0,72}\right), 1043.9 \mathrm{~nm}$ (mode $\left.T E_{10,68}\right)$ appeared at1006.2 nm (Q=12000), $1037.4 \mathrm{~nm}(\mathrm{Q}=18000)$ and $1041.4 \mathrm{~nm}(\mathrm{Q}=14000)$, respectively. Power budget analyses indicate that the power coupled into the waveguide for the modes $T E_{1,72}, T E_{0,72}$ and $T E_{10,68}$ are $36 \%, 42 \%$ and $57 \%$, respectively. The vertical losses account (in average) for about 3\% of the input power escaping through the air layer and 6\% escaping through the oxide layer; the remaining percentage of the input power is radiated laterally. The main reason for not launching only $3 D F D T D$ simulations is that they pushed our computer resources to the limit, both in terms of memory and computation time (3D FDTD simulations took 5 to 7 days to complete). Hence, we will limit our 3D FDTD simulations to a 
few selected cases.

\section{MODIFIED SQUARE RESONATOR WITH THE ADDITION OF AN AIR REGION}

The occurrence of these domains is interesting and to further analyze these domains we envisaged the following scheme: we replaced one of these domains by a rhombic air region (actually a square air region rotated by 45 degrees). This is shown in Fig. 4(a), where the rhombic air region is placed close to the rightmost side of the square. Because of the symmetry of the square resonator, it doesn't make much difference if we place these rhombic air regions adjacent to any of the sides of the square resonator, the main difference is that the coupling efficiency into the waveguide may vary a little bit.

This rhombic region emulates, approximately, a magnetic wall region[24]. The position of the rhombic inclusion (air region) with respect to a given side, on the other hand, changes the spectral resonances of the square resonator. We aimed to analyze the effects of the rhombic inclusion in two modes: $T E_{0,72}$ (mode A) that has "well" ordered domains and $T E_{10,68}($ mode B) with not so "ordered" domains.

Let us consider initially the effects of the addition of the air region in a node position of mode A, replacing it by an air inclusion. We optimized both the position and size (dimension $\mathrm{W}_{3}$ ) of the air rhombic inclusion (the word position refers to the position of the leftmost edge of the inclusion, as shown in Fig. 4(a) It should be mentioned that the optimum values of the size and position of the air rhombic inclusion are highly dependent upon the mode. The optimum values depend on the given mode, because the number of domains depends not only upon the dimensions of the square resonator but also on the mode characteristics (a gross way to understand this is to remember that the number of nodes and peaks in a certain resonator depends upon the electromagnetic resonant mode). In case of modes $\mathrm{A}$ and $\mathrm{B}$, the optimum size of the inclusion is about $1.1 \mu \mathrm{m}$, implying that the size of each domain is about $1.1 \mu \mathrm{m}$.

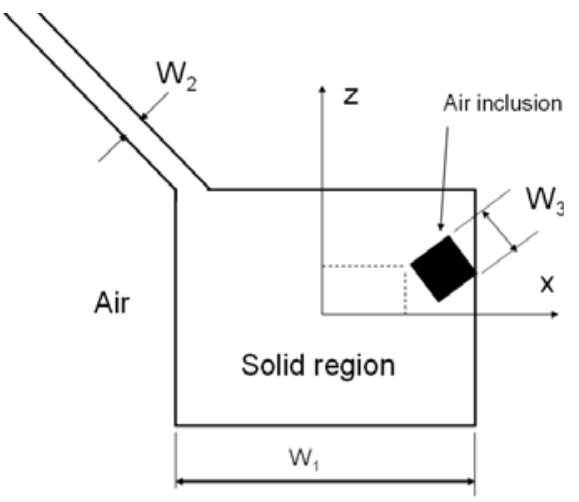

(a)

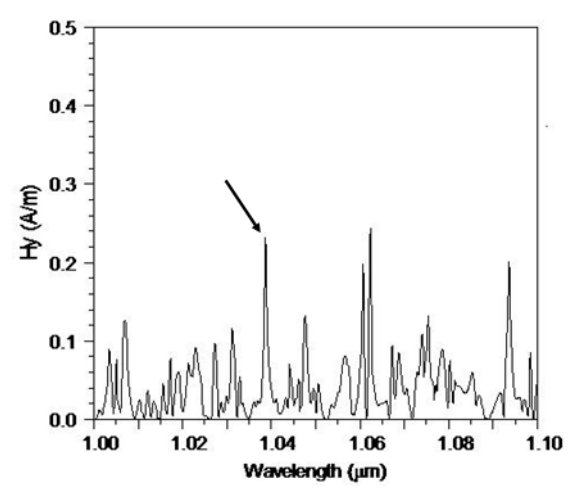

(b)

Fig. 4. Square resonator with an air rhombic section (rotated square region) replacing the domain of mode A (a) schematic, (b) Magnetic field spectrum (Hy) at the center of the waveguide. The rhombic air inclusion is located at $\mathrm{x}=3.45 \mu \mathrm{m}$ and $\mathrm{z}=-2.6 \mu \mathrm{m}$.

Figs 4(b), 5(a) and 5(b) show the $H_{y}$ spectrum for an air inclusion located at $x=3.45 \mu \mathrm{m}$ (the edge of 
the air inclusion touches the rightmost side of the square resonator). The $z$ coordinates in Figs. 4(b), 5(a) and 5(b) are $z=-2.6 \mu \mathrm{m}, z=-0.9 \mu \mathrm{m}$ and $z=2.78 \mu \mathrm{m}$, respectively. These positions were optimized to produce the maximum quality factor for mode $A$ and make the air inclusion to overlap a domain of mode $A$ (there are five similar domains along the $x$-direction. Beginning from the leftmost side of the square resonator, we can observe two half domains and 4 full domains for mode $A$, but the two "central" domains have similar spectral response. For this reason, we present only three spectral responses in the waveguide). In all these Figs., the quality factor of mode $A$ is about 3000 . The main reason for the reduction of the quality factor of this mode is that the introduction of the air inclusion leads to additional radiation losses. The field intensity in the waveguide is stronger when $z=-2.6 \mu \mathrm{m}$ but not much different from the case when $z=2.78 \mu \mathrm{m}$. There is no strong mode selectivity by introducing this air inclusion, since we can clearly see many other modes with similar quality factors. Based upon these results, it seems that the actual size of the domain for mode $A$ is indeed about 1.1 $\mu \mathrm{m}$.

Much more interesting results arise when we place an air inclusion at the domains of mode $B$. The domains for mode $B\left(T E_{10,68}\right)$ are not as well ordered as in the case of mode $A$, as can be observed in Fig. 3(b). Although we obtained similar spectra by either placing the air inclusion closer to the edges of the square resonator or close to the side center, the best result was obtained when we placed the air inclusion close to the center of the rightmost side of the square. The $H_{y}$ field spectrum is shown in Fig. 6(a), where the air inclusion is located at $(x=3.45 \mu \mathrm{m}, z=-0.12 \mu \mathrm{m})$, close to the center of the square. There are several peaks, in the region between $1000 \mathrm{~nm}$ and $1100 \mathrm{~nm}$, with quality factors of about 1000. These peaks can be observed, for example, at $1005.3 \mathrm{~nm}, 1029.9 \mathrm{~nm}$ and $1032.2 \mathrm{~nm}$. However, there is a main peak at $1044 \mathrm{~nm}$ (which shifts to $1043.4 \mathrm{~nm}$ in 3D simulations) with a quality factor of 6600 (2D simulations) and 5600 (3D simulations), which corresponds to the resonant wavelength of mode $T E_{10,68}$.In this case, the introduction of an air inclusion to mode $\mathrm{B}$ was much more selective (a factor of difference of nearly 5:1 between the quality factors of different modes). Since this mode has much higher $\mathrm{Q}$ than the remaining peaks, this laser device will be essentially single-mode over a wide range of pumping power. 


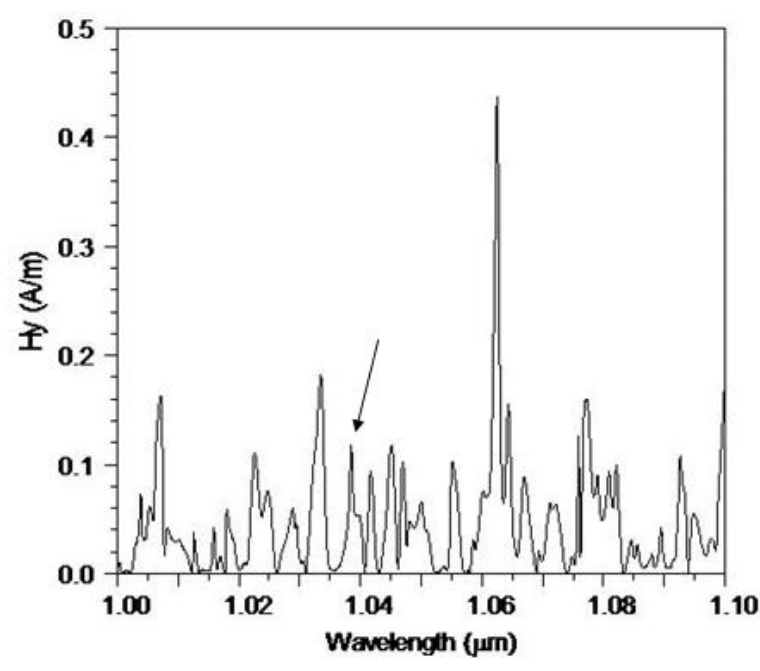

(a)

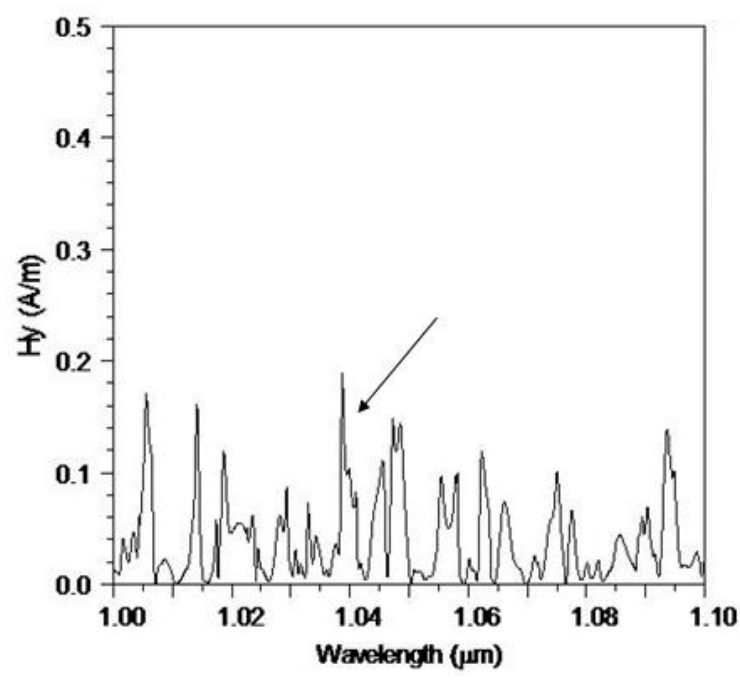

(b)

Fig. 5. Square resonator with an air rhombic section (rotated square region) replacing the domain of mode A: (a) Magnetic field spectrum (Hy) at the center of the waveguide. The rhombic air inclusion is located at $\mathrm{x}=3.45 \mu \mathrm{m}$ and $\mathrm{z}=-0.9 \mu \mathrm{m}$. (b) Magnetic field spectrum (Hy) at the center of the waveguide.

The rhombic air inclusion is located at $\mathrm{x}=3.45 \mu \mathrm{m}$ and $\mathrm{z}=2.78 \mu \mathrm{m}$.

The magnetic field distribution $\left(H_{y}\right)$ at $1044 \mathrm{~nm}$ is shown in Fig. 6(b). We can note that the addition of the air region distorts the magnetic field, but there is resemblance to the field distribution shown in Fig. 3(b). Besides that, the air inclusion is definitely not a perfect magnetic wall: there is some leakage into the air rhombic region. A $3 D$ power budget analysis indicates that about $30 \%$ of the input power is coupled into the waveguide, with about $4 \%$ escaping through air and $8 \%$ through the oxide region, the remaining power is lost laterally. This reduction of power coupling seems to indicate that the radiation losses increased with the introduction of the air region.

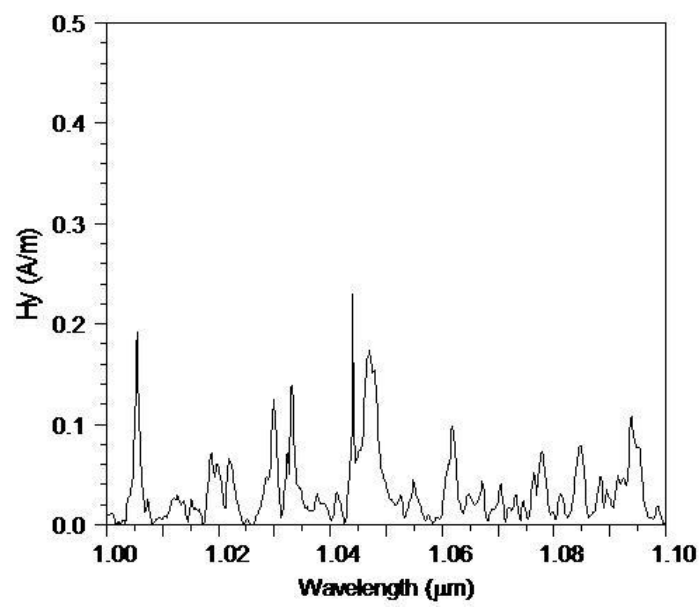

(a)

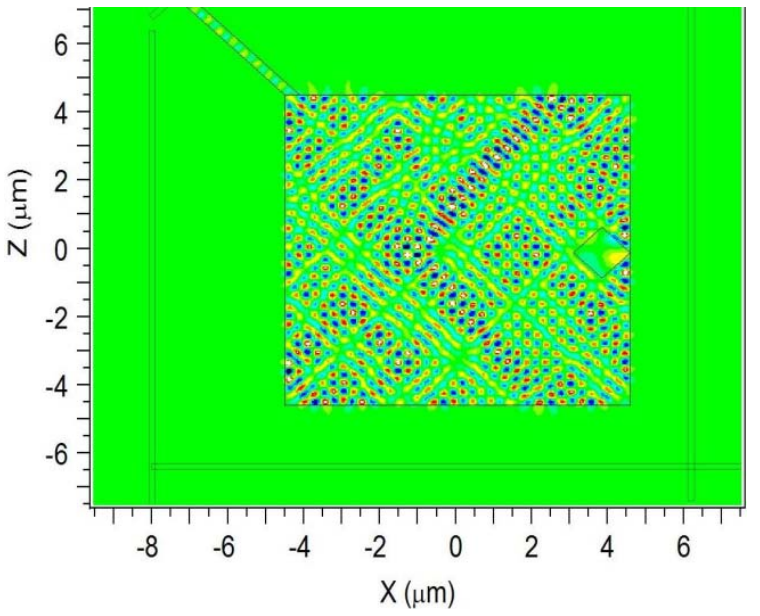

(b)

Fig. 6. Square resonator with an air rhombic section (rotated square region) replacing the domain of mode B: (a) Magnetic field spectrum (Hy) at the center of the waveguide. The rhombic air inclusion is located at $x=3.45 \mu \mathrm{m}$ and $\mathrm{z}=-0.12 \mu \mathrm{m}$. (b) Field distribution at $\lambda=1044 \mathrm{~nm}$.

In Fig. 7(a), we changed the $z$-position of the air inclusion with respect to the optimum position. We can clearly observe that if we displace the edges of the air inclusion, they will be displaced with 
respect to the positions of minimum magnetic field what can lead to additional radiation losses. It is evident that if we displace this air inclusion to another domain of the selected mode, we will return to the situation where the edges of the inclusion match the positions of minimum magnetic field and the quality factor will be high again. However, if we change slightly (hundreds of nanometers) the zposition of the air inclusion, we won't be in this situation and we clearly observe that the quality factor drops with the z-displacement. Based upon Fig. 7(a), we may argue that a displacement from the optimum position of $\pm 100 \mathrm{~nm}$ can be tolerated, leading to a reduction of $\mathrm{Q}$ to about 3500 .

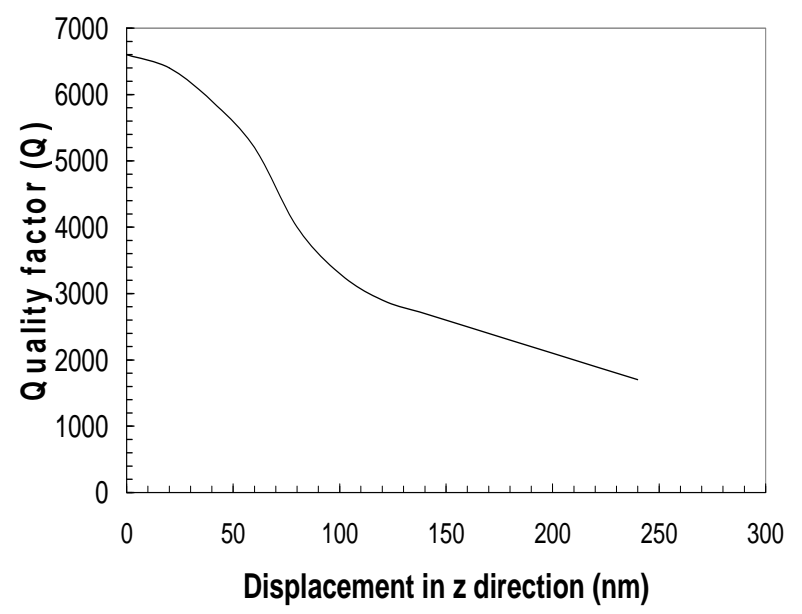

(a)

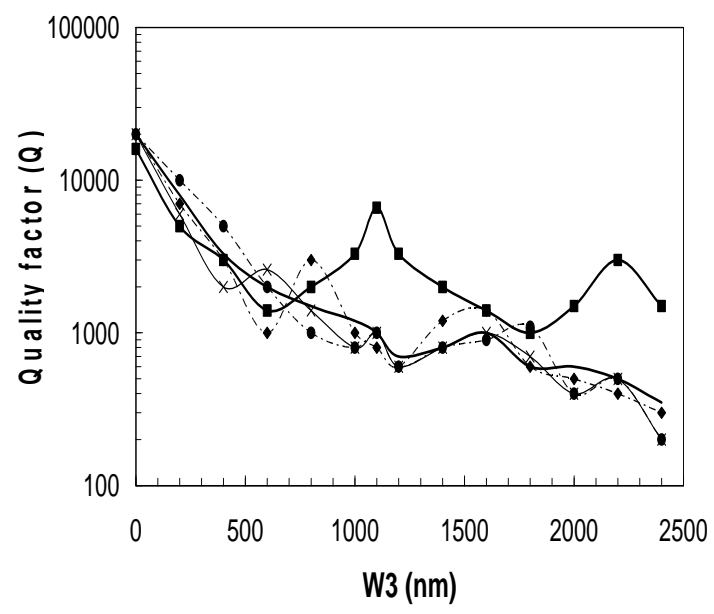

(b)

Fig. 7. Square resonator with an air rhombic section (rotated square region) replacing the domain of mode B: (a) Quality factor (Q) as a function of the displacement of the air inclusion, with respect to the optimum point, along the z-direction. (b) Quality factor (Q) as a function of the size of the air inclusion W3.

In Fig. 7(b), we change the size of the air inclusion $\left(W_{3}\right)$. The effect of the size of the air inclusion on several modes is assessed. The effects on the resonant modes at $1022 \mathrm{~nm}, 1038.1 \mathrm{~nm}, 1043.9 \mathrm{~nm}$ (selected mode), $1045.9 \mathrm{~nm}$ and $1063.2 \mathrm{~nm}$ are represented by the plots with dotted line with triangular markers, solid line with no markers, solid line with squared markers, solid line with xmarkers and dotted line with circular markers, respectively. We can clearly note the oscillations in these plots as will be explained later, but let us concentrate on the plot of solid line with squared markers that represent the effect of the air inclusion on the selected mode. A very small air inclusion has no major effects on the square resonator. As the size of the air inclusion increases, it critically disturbs the points of high magnetic field what leads to increasing radiation losses and reduction of the quality factor of this mode. However, as the size of the air inclusion increases, there will be a point where the edges closely match the points of minimum magnetic field (this happens at approximately $W_{3}=1060 \mathrm{~nm}$ ) and the perturbation to the magnetic field is not so high and leads to a high Q. The same happens when the air inclusion covers four domains (at $W_{3}=2200 \mathrm{~nm}$ ), but at this stage, the introduction of such a large scatterer leads to significant higher radiation losses. This explains the appearance of "oscillations" in the plot. Finally, it should be said that a variation of \pm 100 
$\mathrm{nm}$ in $W_{3}$ can be tolerated by the square resonator. The addition of two air inclusions in the square resonator leads to a lower $\mathrm{Q}$ because of larger radiation losses when two scatterers are introduced.

\section{STEADY-STATE ANALYSIS OF THE MODIFIED SQUARE RESONATOR WITH THE ADDITION OF AN AIR REGION.}

The steady-state response of the modified square resonator with an air inclusion to select mode $T E_{10,68}$ can be obtained by solving the rate equations (4.a) and (4.b) [7], [18], [25], [29], [30]. In the active layer, we have three $7.4 \mathrm{~nm}$ thick $I n_{0.2} \mathrm{Ga}_{0.8} A s$ quantum wells. Since this epitaxially grown layer is primarily targeted for optically pumped sources, we will analyze the response of this laser device to an external laser source (vertically reaching the device). The rate equations that describe the performance of the device are given by [31],

$$
\begin{gathered}
\frac{d N}{d t}=\eta \frac{P_{\text {in }} \lambda_{p}}{h c_{o} V_{a}}-\left(A N+B N^{2}+C N^{3}\right)-\Gamma G(N) S \\
\frac{d S}{d t}=\Gamma G(N) S+\beta B N^{2}-\frac{S}{\tau_{p}}
\end{gathered}
$$

where $N$ is the carrier density, $S$ the photon density, $P_{i n}$ is the pump power and the remaining parameters are summarized in Table 1.

\begin{tabular}{|c|c|c|}
\hline Symbol & Quantity & Typical value \\
\hline$\eta$ & $\begin{array}{l}\text { Absorption ratio of } \\
\text { pump in QW region }\end{array}$ & 0.26 \\
\hline$\lambda \mathrm{p}$ & Pump wavelength & $670 \mathrm{~nm}$ \\
\hline $\mathrm{h}$ & Planck's constant & $6.626 \times 10^{-34} \mathrm{~m}^{2} \mathrm{~kg} / \mathrm{s}$ \\
\hline co & $\begin{array}{c}\text { Free-space speed of } \\
\text { light }\end{array}$ & $299792458 \mathrm{~m} / \mathrm{s}$ \\
\hline$\eta_{\mathrm{a}}$ & Coupling efficiency & 0.3 \\
\hline A & $\begin{array}{l}\text { Linear } \\
\text { recombination rate }\end{array}$ & $2.5 \times 10^{-8} \mathrm{~S}^{-1}$ \\
\hline $\mathrm{B}$ & $\begin{array}{l}\text { Bimolecular } \\
\text { recombination } \\
\text { coefficient }\end{array}$ & $1.6 \times 10^{-16} \mathrm{~m}^{3} / \mathrm{s}$ \\
\hline $\mathrm{C}$ & $\begin{array}{l}\text { Auger nonradiative } \\
\text { recombination rate }\end{array}$ & $5 \times 10^{-41} \mathrm{~m}^{6} / \mathrm{s}$ \\
\hline$\Gamma$ & Confinement factor & 0.22 \\
\hline$\beta$ & $\begin{array}{l}\text { Spontaneous } \\
\text { emission factor }\end{array}$ & $10^{-4}$ \\
\hline
\end{tabular}

TABLE I. PARAMETERS AND ITS VALUES

The pumped active volume $\left(V_{a}\right)$ depends on the parameters of the pump laser beam (spot-size diameter) and the active region thickness. The thickness of the active region is different from the thickness of the active/core layer [30], including mainly the quantum wells and confinement and space barriers. The photon lifetime is given by[25], [31], 


$$
\tau_{p}=\frac{Q \lambda_{l}}{2 \pi c_{o}}
$$

where $\lambda_{l}$ is the emitting wavelength. The gain $G(N)$ for quantum wells can be expressed as [32],

$$
G(N)=v_{g} G_{o} \ln \left(\frac{N}{N_{t r}}\right)
$$

where $v_{g}$ is the group velocity, $G_{o}=1.5 \times 10^{5} \mathrm{~m}^{-1}$ is the gain coefficient and $N_{t r}=1.5 \times 10^{24} \mathrm{~m}^{-3}$ is the transparency carrier concentration.

The final task is to solve the rate equations (3.a) and (3.b). A fourth-order Runge-Kutta method could be employed to solve these equations, but further simplification can be obtained by assuming that, under steady-state, $d S / d t=d N / d t=0$. Once we obtain the steady-state photon density $S$, the output power will be given by [25], [32],

$$
P_{\text {out }}=\eta_{a} \frac{h c_{o}}{\lambda_{l}} S \frac{V_{\text {mode }}}{\tau_{\text {mirror }}}
$$

where $\eta_{a}$ is the coupling efficiency to the ridge, Vmode is the optical mode volume and $\tau_{\text {mirror }}=\tau_{p}$ is the mirror lifetime.

Not all the output power is coupled into the single-mode waveguide. However, finite-difference time-domain (FDTD) simulations can provide us information about the coupling efficiency into the waveguide $\left(\eta_{w g}\right)$. The power coupled into the waveguide is then given by,

$$
P_{w g}=\eta_{w g} P_{o u t}
$$

Note that $P_{w g}$ is the total power coupled into the waveguide.

The threshold values of the laser devices can also be determined by using linear electromagnetic analysis [33], i.e., the threshold is reached when the gain compensates the losses in the cavity. In this sense, the threshold power of the laser devices can be determined given that gain is added to the numerical FDTD method. The main characteristics of the mode are determined by applying Green's formulas to the total field and calculating and balancing the energy in the cavity [33]. 


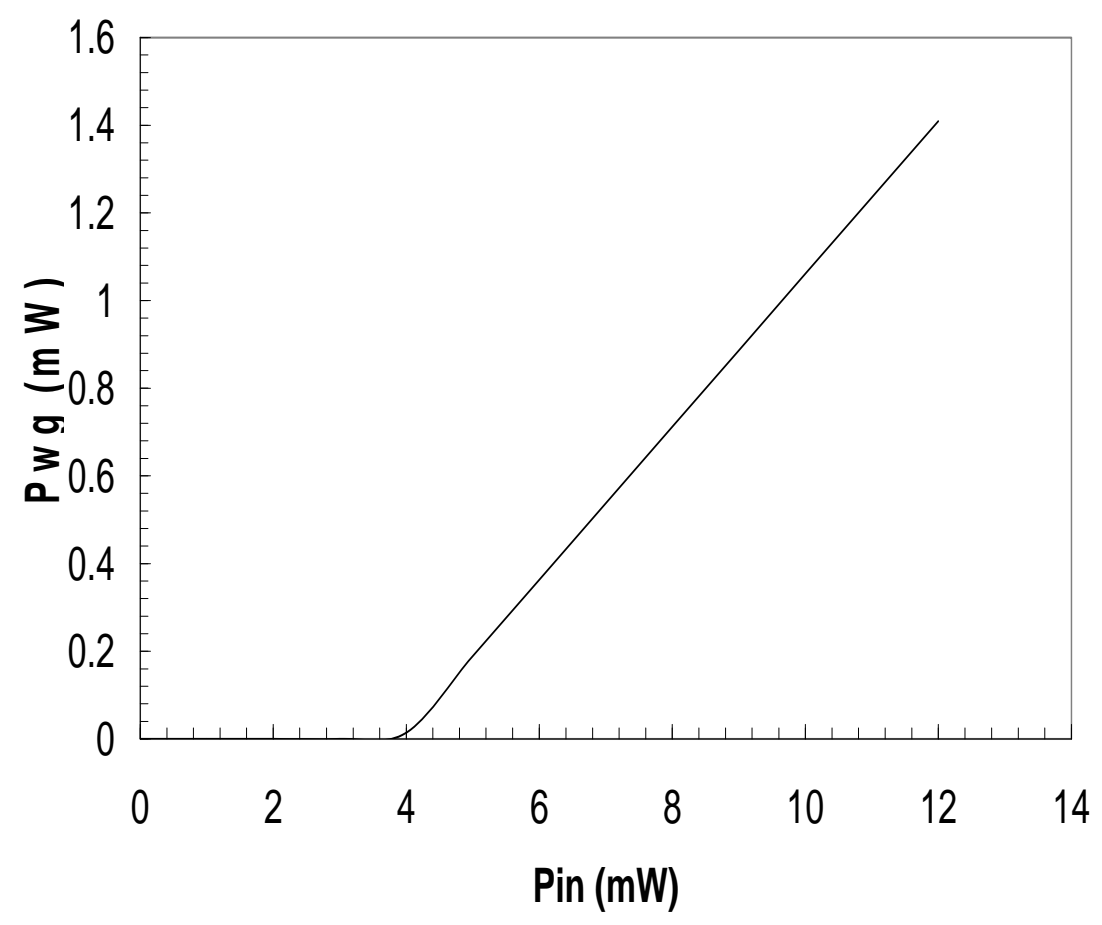

Fig. 8. Power guided into the waveguide.

We assume that the pump beam spot-size diameter is $10 \mu \mathrm{m}$ (typical value that can be reached by our micro-photoluminescence setup). The power guided into the waveguide is shown in Fig. 8. Based upon this analysis, the threshold pump optical power is about $3.9 \mathrm{~mW}$ and we might be able to couple a few $\mathrm{mW}$ into the single-mode waveguide.

\section{CONCLUSIONS}

We have analyzed the modal domains in square resonators. These domains repeat themselves in the large square and are very dependent upon the resonant mode.We estimated the size of the domains by either observing the electromagnetic field distribution or adding an air inclusion to the square resonator. The addition of the air inclusion can lead to quasi single-mode operation over a wide range of wavelengths and over wide range of pumping power.

\section{ACKNOWLEDGMENT}

The author gratefully acknowledges the financial support to this project by the Australian Research Council (ARC). Ziyuan Li acknowledges the UNSW internal fellowship.

\section{REFERENCES}

[1] O. Painter, R. K. Lee, A. Scherrer, A. Yariv, J. D. O’Brien, P. D. Dapkus, “Two-dimensional photonic bandgap defect mode laser, " Science 284, 1819-1821, Jun. 1999.

[2] H. G. Park, J. K. Hwang, J. Huh, H. Y. Ryu, S. H. Kim, J. S. Kim, and Y. H. Lee, " Characteristics of modified singledefect two-dimensional photonic crystal lasers, “ IEEE J. Quantum Electron. 38, 1353-1365, Oct. 2002.

[3] D. S. Song, S. H. Kim, H. G. Park, C. K. Kim, and Y. H. Lee, " Single-fundamental-mode photonic crystal verticalcavity surface-emitting lasers, “ Appl. Phys. Lett. 80, 3901-3903, May 2002.

[4] T. Asano, M. Mochizuki, S. Noda, M. Okano, and M. Imada, " A channel drop filter using a single-defect in a 2D photonic crystal slab: defect engineering with respect to polarization mode and ratio of emission from upper and lower sides," J. Lightwave Technol. 21, 1370-1376, May 2003. 
[5] S. Fan, S. G. Johnson, J. D. Joannopoulos, C. Manolatou, and H. A. Haus, "Waveguide branches in photonic crystals," J. Opt. Soc. Am. B 18, 162-165, Feb. 2001.

[6] C. Seassal, C. Monat, J. Mouette, E. Touraille, B. Ben Bhakir, H. T. Hattori, J. L. Leclercq, X. Letartre, P. RojoRomeo, P. Viktorovitch, "InP bonded membrane photonics components and circuits: toward 2.5 dimensional micronano-photonics,” IEEE J. Sel. Top. In Quantum Electron. 11, 395-407, March /April2005.

[7] H. T. Hattori, H. H. Tan, and C. Jagadish, "Optically pumped in-plane photonic crystal micro-cavity laser arrays coupled to waveguides,” IEEE/OSA J. Lightwave Technol. 26, 1374-1380, Jun. 2008.

[8] R. M. Cazo, C. L. Barbosa, H. T. Hattori, and V. M. Schneider, "Steady-state analysis of a directional square lattice band-edge photonic crystal laser," Microw. Opt. Technol. Lett. 46, 210-214, August 052005.

[9] H. T. Hattori, C. Seassal, X. Letartre, P. Rojo-Romeo, J. L. Leclercq, P. Viktorovitch, M. Zussy, L. di Cioccio, L. El Melahoui, and J. M. Fedeli, "Coupling analysis of heterogeneous integrated InP based photonic crystal triangular lattice band-edge lasers and silicon waveguides," Opt. Express 13, 3310-3322, May 2005.

[10] C. Genet and T. W. Ebbesen, "Light in tiny holes," Nature 445, 39-46, Jan. 2007.

[11] E. Laux, C. Genet, T. Skauli, and T. W. Ebbesen, "Plasmonic photon sorters for spectral and polarimetric imaging," Nature Phot. 2, 161, Feb. 2008.

[12] N. Yu, E. Cubukcu, L. Diehl, M. A. Belkin, K. B. Crozier, F. Capasso, D. Bour, S. Corzine and G. Hofler, "Plasmonic quantum cascade laser antenna, “Appl. Phys. Lett. 91, 173113, Oct. 2007.

[13] A. Minovich, H. T. Hattori, I. McKerracher, H. H. Tan, D. N. Neshev, C. Jagadish and Y. S. Kivhsar, "Enhanced transmission of light through periodic and chirped lattices of nanoholes, "Opt. Comm. 282, 2023-2027, May 2009.

[14] M. Fujita, A. Sakai, and T. Baba, "Ultra-small and ultra-low threshold GaInAsP-InP microdisk injection laser- design, fabrication, lasing characteristics and spontaneous emission factor, “IEEE J. Sel. Top. Quantum Electron. 5, 673-681, May/Jun.1999.

[15] A. F. J. Levi, R. E. Slusher, S. L. McCall, J. L. Glass, S. J. Pearton, and R. A. Logan, "Directional light coupling from microdisk lasers, “Appl. Phys. Lett. 62, 562-563, Feb.1993.

[16] S. V. Boriskina, T. M. Benson, P. D. Sewell, and A. I. Nosich, "Directional emission, increased free spectral range, and mode Q-factors in 2-D wavelength-scale optical microcavity structures, “ IEEE J. Sel. Top. Quantum Electron. 12, 1175-1182, Nov./Dec. 2006.

[17] H. T. Hattori, C. Seassal, E. Touraille, P. Rojo-Romeo, X. Letartre, G. Hollinger, P. Viktorovitch, L. DiCioccio, M. Zussy, L. El Melhaoui, and J. M. Fedeli, "Heterogeneous integration of microdisk lasers on silicon strip waveguides for optical interconnects, “IEEE Phot. Technol. Lett. 18, 223-225, Jan. 2006.

[18] H. T. Hattori,"Analysis of optically pumped equilateral triangular microlasers with three mode-selective trenches," Appl. Optics 47, 2178-2185, Apr. 2008.

[19] S. Ando, N. Kobayashi, and H. Ando, "Triangular-facet lasers coupled by a rectangular optical waveguide, “ Jpn. J. Appl. Phys. 36, L76-L78, Feb. 1997.

[20] Y. Z. Huang, W. H. Guo, and Q.M.Wang, “Analysis and numerical simulation of eigenmode characteristics for semiconductor lasers with an equilateral triangle micro-resonator, “IEEE J. Quantum Electron. 37, 100-107, Jan. 2001.

[21] Y. Z. Huang, W. H. Guo, L. J. Yu, and H. B. Lei“" Analysis of semiconductor microlasers with an equilateral triangle resonator by rate equations, “IEEE J. Quantum Electron. 37, 1259-1264, Oct. 2001.

[22] W. H. Guo, Y. Z. Huang, Q. Y. Lu, L. J. Yu, “ Mode quality factor based on far-field emission for square resonators, “ IEEE Phot. Technol. Lett. 16, 479-481, Feb. 2004.

[23] H. T. Hattori, D. Liu, H. H. Tan, and C. Jagadish, "Large square resonator laser with quasi-single-mode operation, “ IEEE Phot. Technol. Lett. 21, 359-361, Mar. 2009.

[24] W. H. Guo, Y. Z. Huang, Q. Y. Lu, and L. J. Yu, “Whispering-gallery-like modes in square resonators,” IEEE J. Quantum Electron. 29, 1106-1110, Sep. 2003.

[25] H. T. Hattori, "Modal analysis of one-dimensional nonuniform arrays of square resonators," J. Opt. Soc. Am. B 25, 1873-1881, Nov. 2008.

[26] W. H. Guo, Y. Z. Huang, Q. Y. Liu and L. J. Yu, "Modes in square resonators,” IEEE J. Quantum Electron. 39, 15631566, Dec. 2003.

[27] D. Ohnishi, T. Okano, M. Imada, and S. Noda, "Room temperature continuous wave operation of a surface-emitting two-dimensional photonic crystal diode laser, " Opt. Express 12, 1562-1568, Apr. 2004.

[28] W. Chang, A. Ankiewics, J. M. Soto-Crespo and N. Akhmediev, "Dissipative soliton resonances," Phys. Rev. A 78, 023830,2008

[29] N. Djellali, I. Gozhyk, D. Owens, S. Lozenko, M. lebental, J. Lautru, C. Ulysse, B. Kippelen, and J. Zyss, "Controlling the directional emission of holey organic microlasers," Appl. Phys. Lett. 95, 101108, 2009.

[30] Fullwave 4.0 RSOFT design group, 1999, http://www.rsoftdesign.com

[31] H. Altug and J. Vuckovic, "Photonic crystal nanocavity laser array," Opt. Express 13, 8819-8828, Oct. 2005.

[32] L. A. Coldren and S. W. Corzine, Diode Lasers and Photonic Integrated Circuits, 1st ed. New York: Wiley, 1995.

[33] E. I. Smotrova, V. O. Byelobrov, T. M. Benson, J. Ctyroky, R. Sauleau, and A. I. Nosich, "Optical theorem helps understand thresholds of lasing in microcavities with active regions,” IEEE J. Quantum Electron. 47, 20-30, 2011. 